\title{
Finding the Shortest Course of a Ship Based on Reinforcement Learning Algorithm
}

\author{
Kunihiko MITSUBORI", Takeshi KAMIO ${ }^{* *}$ and Takahiro TANAKA ${ }^{* * *}$
}

\begin{abstract}
Recently, great attention has been paid to the reinforcement learning (RL) algorithm in the fields of the artificial intelligence and the machine learning, as a tool to solve a class of the optimization problem. We try to construct the RL framework to find the shortest course of a ship in the following fundamental situations:

(A) A ship goes on a restricted sea-area with the strong tidal current, such as the Kurushima strait.

(B) Two ships go on a sea-area with no tidal current while each of them avoids the collision with the other. $Q$-learning algorithm, which is representative of the $\mathrm{RL}$ algorithm, is combined with the ship's motion equations through the quantization of their variables. Finally, the effectiveness of our framework is demonstrated with the model of the sea-area.
\end{abstract}

Keywords : artificial intelligence, reinforcement learning, course setting, collision avoidance

\section{Introduction}

This paper discusses how to find the shortest course of a ship. The ship is a dynamical system which has the following characteristics:

i) There is no way to brake and to go backward effectively.

ii) The attitude is unstable at a low speed.

iii) The dynamics is nonlinear.

This fact means that finding the shortest course is important but difficult. We treat finding the shortest course in the following situations as the fundamental problems:

(A) A ship goes on a restricted sea-area with the strong tidal current, such as the Kurushima strait.

(B) Two ships go on a sea-area with no tidal current while each of them avoids the collision with the other.

As a useful tool to solve these problems, we focus on the reinforcement learning (RL) algorithm ${ }^{(1)}$. The $\mathrm{RL}$ is one of the most important learning algorithms in the fields of the artificial intelligence and the machine learning. It has been applied to the optimization problems, such as the dynamical behavior of a double pendulum and so on ${ }^{(1)}$. In this algorithm, the learner optimizes a sequence of the action through the repeat of trial and error. This repeat is executed according to natural passage of time. This algorithm can be developed into the optimization of the cooperative actions between the plural learners ${ }^{(2)}$.

Next we show the reason why we use the RL algorithm to optimize the maneuvering motion of a ship. Our target is to find the shortest courses of many ships in the realistic situation. In order to approach this target, we consider the situations (A) and (B) as the fundamental problems. Therefore, we have to construct the framework appropriate for solving them.

In the field of the navigation, the use of the dynamic programming in the situation (A) is well known as a technique in the weather routings. On the other hand, the situation (B) has been treated in the maneuvering simulation and the automatic operation, where the course has been given as "a guideline" which the ship should trace and the procedures to avoid the collision between the ships have been $\begin{array}{lllllllll}d & i & s & c & u & s & s & e & d\end{array}$

* Member: Japan Coast Guard Academy, Kure-shi, Hiroshima, 737-8512. mitubori@jcga.ac.jp

** Non-member: Hiroshima City University, Hiroshima-shi, Hiroshima, 731-3194. kamio@im.hiroshima-cu.ac.jp

*** Member: Japan Coast Guard Academy, Kure-shi, Hiroshima, 737-8512. takahiro@jcga.ac.jp 


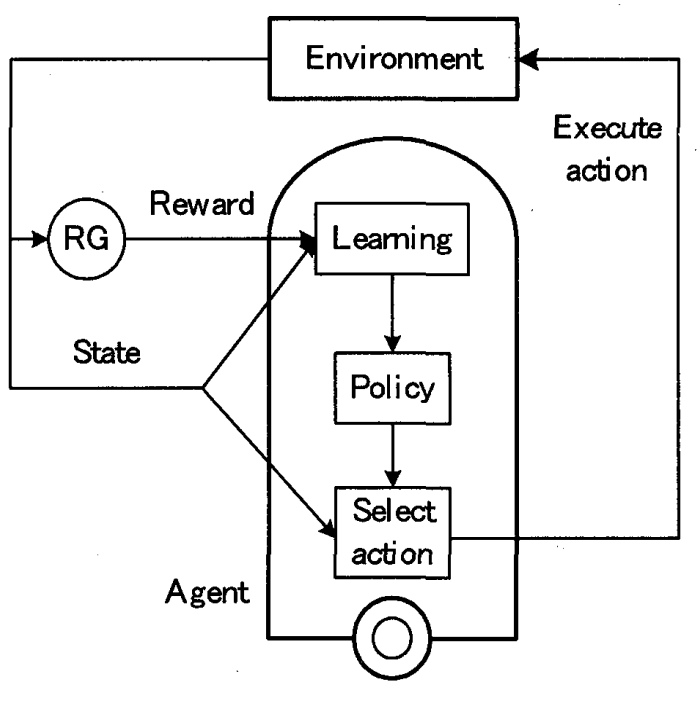

Fig. 1 The keywords of the RL algorithm.

However, these discussions do not include the optimality of the course and do not reflect the interaction between the maneuvering actions of the navigators.

In the field of the artificial intelligence, reference (3) has discussed the RL in maneuvering motion of a ship without the tidal current effect, but nobody has discussed that with the tidal current effect yet (i.e., the situation (A)). The paper has also discussed the RL to avoid the collision between two ships. However, one ship always goes straight and it does not learn the maneuvering motion. The other ship learns the maneuvering motion to avoid the collision with the ship. This RL does not reflect the interaction and has the only single learner. Since the interaction is essential in the situation (B), it should be reflected to find the shortest course. Therefore, reference (3) is not sufficient to construct the framework to achieve our target.

From these backgrounds, we try to construct the RL framework to find the shortest course in the fundamental problems. For the situation (A), we add the tidal current effect to KT model. For the situation (B), we utilize the $R L$ algorithm for "the multi-agents ${ }^{(4) " ~}$ (i.e., the plural learners) to reflect the interaction between the maneuvering actions of the navigators. Such framework enables each ship to learn the maneuvering motion to avoid the collision with the other. This paper is the first example that applies the RL algorithm for the multi-agents to the optimization of the cooperative maneuvering motions between the ships. The effectiveness of our framework is demonstrated with the model of the sea-area in the situations (A) and (B).

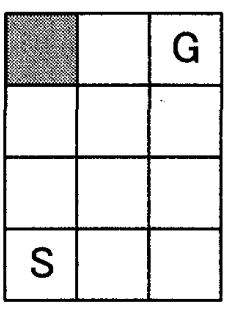

The grid number

\begin{tabular}{|c|c|c|}
\hline 9 & 10 & 11 \\
\hline 6 & 7 & 8 \\
\hline 3 & 4 & 5 \\
\hline 0 & 1 & 2 \\
\hline
\end{tabular}

Fig. 2 The stage of the example problem.

\section{Outline of the RL Algorithm}

This section introduces the outline of the RL algorithm ${ }^{(1)}$. Fig. 1 shows its keywords. In this algorithm, the learner is called agent. The RL problem consists of the agent and the other part which is called environment. State is the variable to express the situation of the environment. Action is the influence of the agent on the environment. Reward is a scalar value to indicate the desirability of each state. The reward is assigned to the state by the reward generator (RG). The agent selects an action in each state according to the rule which is called policy. The agent executes the action and the action updates the state. After this update, the agent receives the reward. The reward reinforces the agent's policy.

We use $Q$-learning algorithm ${ }^{(1)(5)}$, which is representative of the RL algorithm. It can be applied to the optimization problem which is formulated as the finite Markov Decision Process (MDP). Although the original $Q$-learning algorithm has been proposed for the single agent, it can be easily expanded into $n$-agents version. Therefore, we explain $n$-agents version of $Q$-learning algorithm here. Let $k$ denote the agent number hereafter. Each of state and action is specified by integer. Letting $s_{k}$ denote the state number, $s_{k}$ is an element of a finite set $S$ (i.e., $s_{k} \in S$ ). Also, letting $a_{k}$ denote the action number, $a_{k}$ belongs to a finite set $A$ (i.e., $a_{k} \in A$ ). Let $Q_{k}\left(s_{k}, a_{k}\right)$ denote the estimate of the expected reward of $a_{k}$ in $s_{k}$. The validity of $Q_{k}\left(s_{k}\right.$, $a_{k}$ ) is improved by the learning. The $k$-th agent selects $a_{k}$ in $s_{k}$ according to a policy and executes the action specified by $a_{k}$. After the environment is influenced by the action, the $k$-th agent gets a new state number $s_{k}{ }^{\prime}$ from the environment and receives the reward $r_{k}$ which depends on $s_{k}{ }^{\prime}$. Each agent has the corresponding sub-terminal state. If all the agents achieve their sub-terminal states, we regard this situation as the terminal state for the RL problem. Using $a_{k}, s_{k}, r_{k}, s_{k}{ }^{\prime}$ 


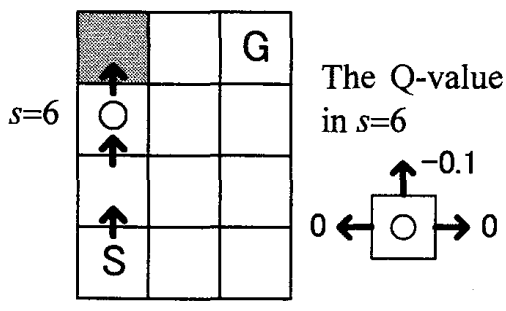

The first trial

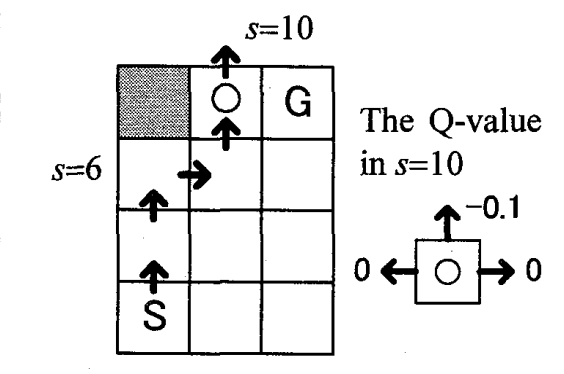

The second trial

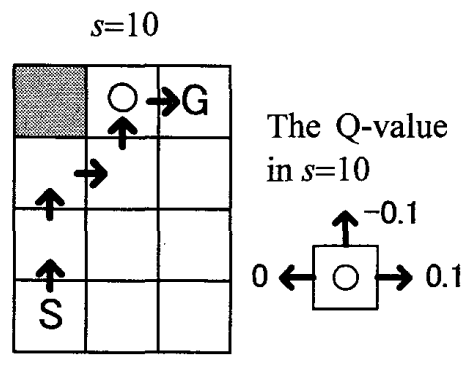

The third trial

Fig. 3 The learning process of the RL.

and $Q_{k}\left(s_{k}, a_{k}\right), n$-agents version of $Q$-learning algorithm is described as follows.

n-agents version of $Q$-learning algorithm:

1. Initialize $Q_{k}\left(s_{k}, a_{k}\right)$ for all available $s_{k}$ and $a_{k}$.

2. Repeat from step a) to step c).

a) Initialize the environment which is the part of the $\mathrm{RL}$ problem except the agent.

b) Repeat from step i) to step v).

i) Select $a_{k}$ by applying a policy to $Q_{k}\left(s_{k}, a_{k}\right)$ in the given $s_{k}$.

ii) Execute the action specified by $a_{k}$.

iii) Get $s_{k}{ }^{\prime}$ from the environment and receive $r_{k}$.

iv) Update $Q_{k}\left(s_{k}, a_{k}\right)$ by

$$
\begin{aligned}
& Q_{k}\left(s_{k}, a_{k}\right) \leftarrow \\
& \quad\left(1-\alpha_{k}\right) Q_{k}\left(s_{k}, a_{k}\right)+\alpha_{k}\left\{r_{k}+\max _{a_{k}^{\prime}} Q_{k}\left(s_{k^{\prime}}{ }^{\prime}, a_{k}{ }^{\prime}\right)\right\},
\end{aligned}
$$

where $\alpha_{k}$ is the learning rate.

v) $s_{k} \leftarrow s_{k}{ }^{\prime}$.

c) Stop the repeat from step i) to step v), if all the agents achieve their sub-terminal states (i.e., the terminal state for the RL problem).

This algorithm is the same as the original version if $n$ $=1$. In this case, we drop the subscript $k$ from $a_{k}, s_{k}, r_{k}$, $s_{k}{ }^{\prime}, Q_{k}\left(s_{k}, a_{k}\right)$ and $\alpha_{k}$ for the sake of simplicity.

In order to explain learning process, we consider a simple shortest path problem for the single agent (i.e., $n=1$ ). Fig. 2 shows the stage of this problem. We refer to the unit square in the stage as the grid. Each grid is numbered. The grid number is utilized as the quantized position. In this problem, the grid number can be identified with the state number. This stage is the environment for this agent and is also the set of the states. This stage has four kinds of grids: normal grid (white), no-entry grid (light-gray), start grid ("S"), and goal grid ("G"). The agent is permitted to enter all grids except the no-entry grids. The agent can move to the up, the right, or the left neighboring gird. Therefore, the agent can select one of these movements as the action. The movements of the agent to the up, the right, and the left are assigned "0", "1", and "2", respectively. These integers are the action numbers in this problem. Moreover, $Q(s, a)$ represents the effectiveness (i.e., value) of the movement (i.e., action) in the gird (i.e., state). Although this problem can be also solved by the dynamic programming, we try to apply $Q$-learning algorithm to this problem according to the following framework:

- The environment is initialized by placing the agent on the start grid "S". The first action at "S" is randomly selected from the movements to the up and the right so that the agent will not escape from the stage.

- In the case of the single agent, the sub-terminal state is identified with the terminal state. The terminal state is the arrival at the goal grid " $G$ ", the entry to the no-entry grid, or the escape form the stage. We refer to the passage period from "S" to the terminal state as a trial.

- As the policy, we employ the greedy policy. In this case, the agent selects the action which satisfies the condition that $Q(s, a)$ has the highest value in the state. If the plural actions satisfy the above condition, the agent selects the action which has the smallest action number.

- The reward $r$ depends on the grid in which the agent is. If the agent is in "S", in the normal grids, in "G", in the no-entry grids, and out of the stage, it receives $r=0,0,1,-1$, and -1 , respectively.

Fig. 3 illustrates the learning process, when the learning rate $\alpha$ is fixed at 0.1 . Here, let us assume that the agent selects the movement to the up as the first action in each trial by chance. As a result, the agent moves along the arrows shown in this figure. In the first trial, 
the agent continues to select the movement to the up (i.e., $a=0$ ) and enters the no-entry grid (i.e., 9th grid) by the third movement. The agent receives $r=-1$ right after this movement, and then returns to "S". Since this reward is used to accumulate the value of the movement to the up (i.e., $a=0$ ) in the 6 th grid (i.e., $s=6), Q(6,0)=-0.1$ is given by the equation (1). In the second trial, the agent moves to the 6 th grid like the first trial, but it does not arrive at the 9th grid. This is because the movement to the up has the smallest value of all the movements in the 6th grid through the first trial. Therefore, the agent must move to either the right (i.e., $a=1$ ) or the left (i.e., $a=2$ ). According to the framework, the agent passes through the 7 th grid, and then arrives at 10th grid. Since the agent in the 10th grid moves to the up, it goes out of the stage. Thus, the agent receives $r=-1$ right after this movement, and then returns to "S". As a result, $Q(10,0)=-0.1$ is given In the third trial, the agent moves to the 10th grid like the second trial, and then must move to either the right or the left. According to the framework, the agent arrives at "G". Therefore, the agent receives $r=1$ right after this movement, and then returns to "S". As a result, $Q(10,1)=0.1$ is given. After the sufficient repeat of such trials, the distribution of the $Q(s, a)$ converges. The shortest path is derived by applying the greedy policy to this distribution. In order to avoid that the agent is trapped in the undesirable path, the combination of the greedy policy and the random selection of the action is often used.

\section{Model of the Maneuvering Motion of a Ship}

We employ a response model, which is often called "KT model", with the tidal current effect. Of course, even if we employ more precise model of the maneuvering motion, we can discuss the $\mathrm{RL}$ algorithm in a manner similar to this paper. Fig. 4 shows the model of the maneuvering motion of a ship in a bird's eye view. In this figure, $O_{S}$ is the center in turning the head of the ship, and $\phi$ is the angle of the head. The location of the ship is represented by that of $O_{S} . L$ is the length from the head to the tail. For the sake of simplicity, let $L$ be divided into two equal parts by $O_{S}$. $\mathbf{v}_{0}$ is the forward velocity vector of this ship and $V_{0}$ denotes the magnitude of $\mathbf{v}_{0} . \delta$ is the rudder angle. The tidal current effect generates the shift of $O_{S}$ and the

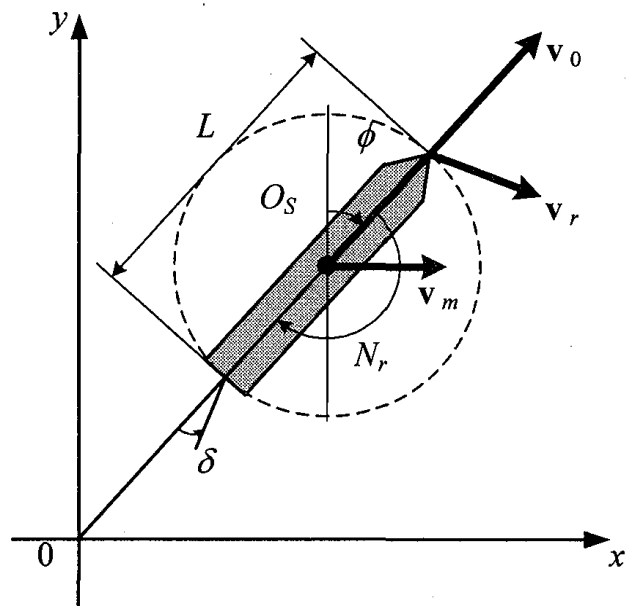

Fig. 4 The model of maneuvering motion of a ship.

Table 1 The principal dimensions of KOJIMA.

\begin{tabular}{|l|l|}
\hline $\begin{array}{l}\text { Dimensionless } \\
\text { maneuverability index }\end{array}$ & $K_{0}=1.310, T_{0}=1.085$ \\
\hline Length & $L=107[\mathrm{~m}]$ \\
\hline Inertia moment in the turn & $I=2.556 \times 10^{5}$ [tons $\left.\mathrm{m}\right]$ \\
\hline Draft & $d=4.4[\mathrm{~m}]$ \\
\hline
\end{tabular}

additional turn of head. They are expressed by two velocity vectors $\mathbf{v}_{m}$ and $\mathbf{v}_{r}$, respectively. Also, let $N_{r}$ denote the moment which makes the head turn. $N_{r}$ is generated by $\mathbf{v}_{r}$. The dynamics of the maneuvering motion is described by the following equations:

$$
\begin{aligned}
& \dot{\phi}=\Phi, \quad T \dot{\Phi}+\Phi=K \delta+\frac{T}{I} N_{r}, \\
& \dot{x}=V_{0} \sin \phi+v_{m x}, \quad \dot{y}=V_{0} \cos \phi+v_{m y},
\end{aligned}
$$

where $\mathbf{v}_{m}=\left(v_{m x}, v_{m y}\right)^{T}$ and $I$ is the inertia moment in the turn of the head. $K$ and $T$ are the parameters to characterize the maneuver performance of the ship in still-water. Their units are [1/s] and [s], respectively. Their values are given by $K=K_{0} /\left(L / V_{0}\right)$ and $T=T_{0} \times$ $\left(L / V_{0}\right)$, where $K_{0}$ and $T_{0}$ are the dimensionless parameters. Each ship has an individual pair of the values of $K_{0}$ and $T_{0}$. We fix the parameters $K_{0}, T_{0}, L$, and $I$ at the corresponding values of the patrol vessel KOJIMA in Japan Coast Guard. Table 1 shows the principal dimensions of KOJIMA. We also fix $V_{0}$ at a certain value which is less than KOJIMA's maximal forward velocity $20[\mathrm{knots}]=10.29[\mathrm{~m} / \mathrm{s}]$ in each later section. $\delta$ is an adjustable parameter.

These motion equations are combined with the learning algorithm through the quantization of the variables. In order to quantize the position $(x, y)$, we make use of the grids, as shown in the previous 


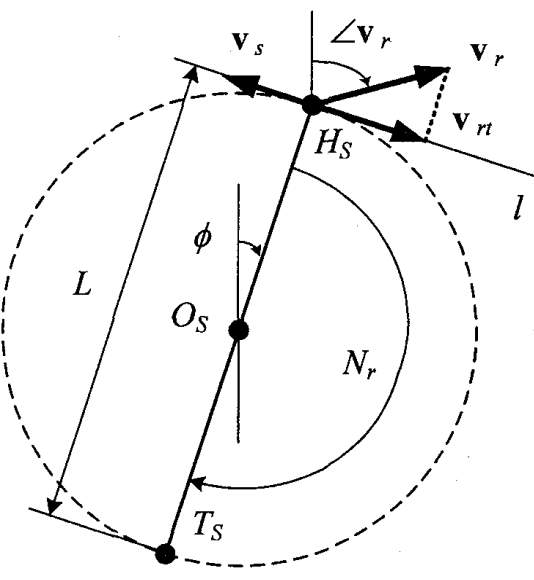

Fig. 5 The moment which makes the head turn.

section. In each later section, we construct a model of the sea-area by the grid. We fix the side length of the grid at $L$ for the sake of simplicity. If the side length is larger than $L$, the generation of $N_{r}$ is not well described. On the other hand, as long as $L$ is $m$ times the side length ( $m$ is a natural number), the calculation of the tidal current effect can be discussed in a similar way to this paper. However, large $m$ causes the increase of the computational cost.

Each grid may have the vector to express the tidal current. Hereafter, we refer to this vector as the tidal current vector. The tidal current vector is uniform in a grid. The calculation procedures of $\mathbf{v}_{m}$ and $\mathbf{v}_{r}$ depend on how the ship-body overlaps with the grids geometrically. Because the side length of the grid is $L$, the overlap situations are classified into the following three cases: I) the ship-body is in a grid, II) the ship-body overlaps with two grids, and III) the ship-body overlaps with three grids. In each case, $\mathbf{v}_{m}$ and $\mathbf{v}_{r}$ are calculated by the tidal current vectors which influence the head and tail of the ship. We next explain the calculation of $N_{r}$ (see Fig. 5). In this figure, $H_{S}$ denotes the head of the ship and $T_{S}$ denotes the tail of the ship. $l$ is the tangent line to the head's locus at $\phi$, $\mathbf{v}_{r t}$ is the projection of $\mathbf{v}_{r}$ along $l$, and $\mathbf{v}_{s}$ is the velocity vector of the head in the turn. We consider that $N_{r}$ is generated by the relative velocity vector between $\mathbf{v}_{r t}$ and $\mathbf{v}_{s} \cdot N_{r}$ is a function of $\left(\mathbf{v}_{r t}-\mathbf{v}_{s}\right)$. We calculate $N_{r}$ by the equation shown in reference (6) and we fix the draft at the value in Table 1 . Also, $N_{r}$ is set to zero forcibly if there is not the tidal current at all or if the head of the ship turns faster than the tidal current.

In the section 4 , we discuss the learning algorithm for a ship using this model. In the section 5 , we also

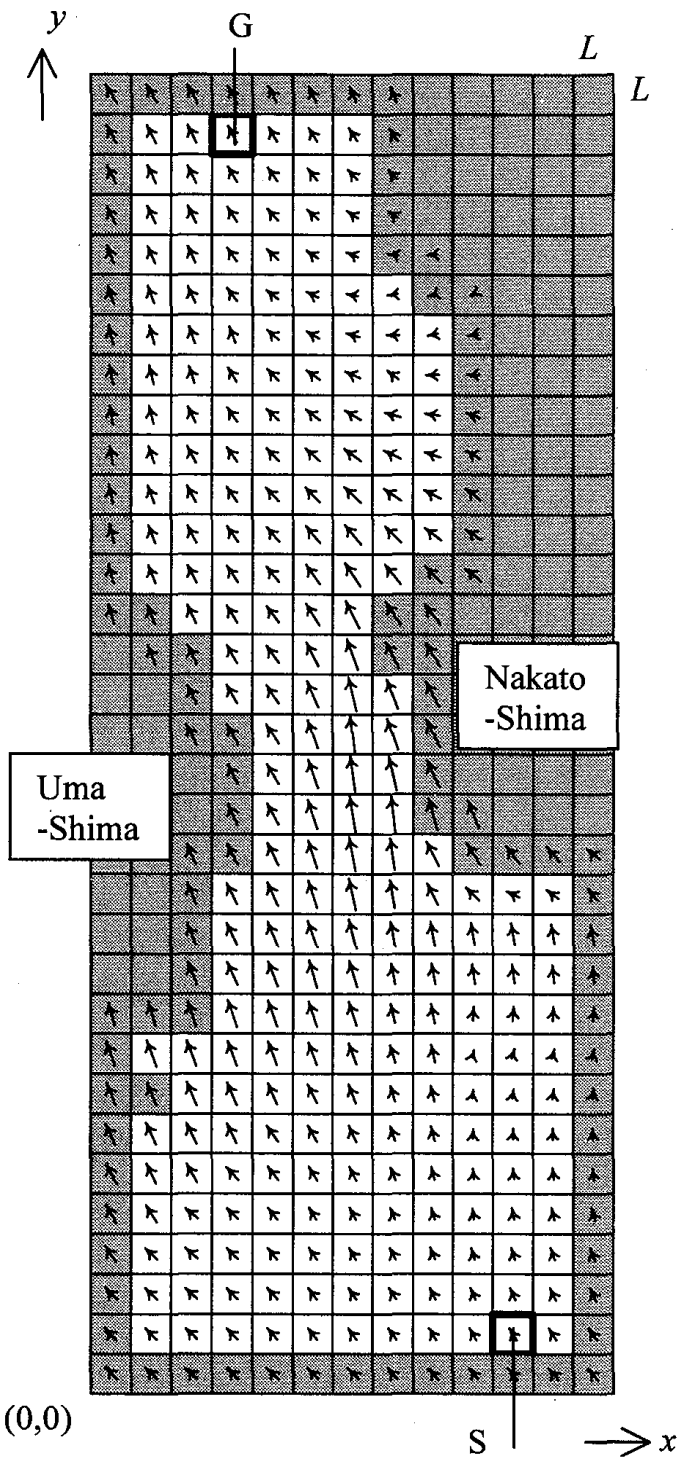

Fig. 6 The model of the sea-area in the situation (A).

discuss the learning algorithm for two ships. We assume that both ships have the identical dynamics which is described by the equation (2) and the common parameter values as shown in Table 1 . Let $k$ denote the ship number and let the variables $\left(\phi_{k}, \Phi_{k}, x_{k}\right.$, $\left.y_{k}\right)$ denote $(\phi, \Phi, x, y)$ in the $k$-th ship. The rudder angle $\delta_{k}$ is an adjustable parameter in the $k$-th ship.

\section{RL Algorithm to Find the Shortest Course in the Situation (A)}

This section discusses the RL algorithm to find the shortest course in the situation (A) and its calculation results. Fig. 6 shows the model of the sea-area. It is based on the chart of tidal streams in Kurushima strait by Japan Coast Guard ${ }^{(7)}$. This model is the sea-area between Uma-shima and Nakato-shima in this strait and it corresponds to the case where this strait has the 


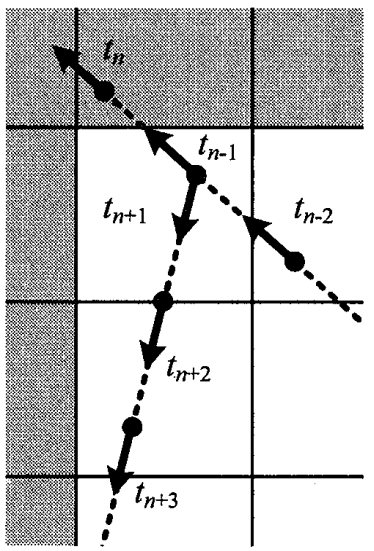

Fig. 7 Escape from the no-entry grid.

maximum north-going stream. It consists of four kinds of the grids defined in the section 2. The no-entry grids represent the obstacles (i.e., the land and the shoal) and the boundary of the sea-area where the ship is permitted to move. The arrow in each grid indicates the tidal current vector. The maximal magnitude of the tidal current vector is $7.4[\mathrm{knots}]=3.81[\mathrm{~m} / \mathrm{s}]$.

We use the single agent (i.e., $n=1$ ) to control the rudder angle of the ship. The aim of the learning algorithm here is finding the shortest course for the ship in the sense of the total number of grids which the ship passes through. We apply the $Q$-learning algorithm to this problem according to the following framework. Note that this framework differs widely from the framework for a simple shortest path problem in the section 2 .

- The environment is represented by the variables $(\phi, \Phi, x, y)$. A point in the space $(\phi, \Phi, x, y)$ corresponds to the state $s$ through the quantization of this space.

- The environment is initialized by setting these variables as follows: $(x, y)$ is set to the center of "S", $\phi$ is randomly selected from a given initial range $I_{0}$ and $\Phi$ is set to zero. The terminal state is the arrival at " $\mathrm{G}$ " or the forcible initialization of the environment which is explained later.

- In order to select the action number, we employ the $\varepsilon$-greedy policy. That is to say, while $a$ is randomly selected from the finite set $A$ with a given small probability $\varepsilon, a$ is also selected by the greedy policy with the probability $(1-\varepsilon)$.

- The movement by the executed action corresponds to 1 time-step of the numerical integration of the motion equations. If the ship enters the no-entry grid, we make it escape from the situation as follows (see Fig.7). If the ship is in the no-entry grid at the time $t_{n}$, the four variables $(\phi$, $\Phi, x, y)$ at $t_{n+1}$ are determined according to the following procedures.

1. Return the ship to the position $(x, y)$ at $t_{n-1}$.

2 . Select $\phi$ randomly from $[0,2 \pi]$ under the condition that the ship must not enter the no-entry grids that neighbor the grid including this $(x, y)$.

3. Set $\Phi$ to zero.

- The reward $r$ depends on the grid in which the ship is. If the ship is in " $G$ ", the no-entry grids and the others, then the agent receives $r=1, r=-1$ and $r=0$, respectively. Also, if the head of the ship is turned over $2 \pi$ in a time-step, the agent receives $r=-1$ and the environment is forcibly initialized to prevent the situation such that the ship keeps on turning the head.

Furthermore, we fix the ship's forward velocity $V_{0}$, the learning rate $\alpha$, the initial angular range $I_{0}$, and the probability $\varepsilon$ at the following values:

$$
\begin{aligned}
& V_{0}=12[\mathrm{knots}]=6.17[\mathrm{~m} / \mathrm{s}], \\
& \alpha=0.3, I_{0}=[-0.2 \pi, 0.0][\mathrm{rad} .], \varepsilon=2.0 \times 10^{-5} .
\end{aligned}
$$

The maximal magnitude of the tidal current vector is almost equal to $62 \%$ of $V_{0}$. For the quantization of the space $(\phi, \Phi, x, y)$, we divide $\phi \in[0,2 \pi]$ into twelve equal parts and $\Phi$ into two parts based on its sign. Also, for the division of the subspace $(x, y)$, we make use of the grids. In the model of the sea-area, the total number of the grids is $13 \times 33=429$ and the space $(\phi, \Phi$, $x, y)$ is divided into $12 \times 2 \times 429=10296$-subspaces Each of them can be classified by the state number $s \in S=\{0,1, \ldots, 10295\}$. In the numerical integration, we use the fourth-order Runge-Kutta method. The size of the time-step is $1 / 35$ of the time for the ship to go straight for the distance $L$. For the selection of the rudder angle $\delta$ [deg.], we prepare the five kinds of the values $\{0,10,-10,20,-20\}$ and they correspond to the action numbers $\{0,1,2,3,4\}$, respectively.

Next, we show the calculation results of the courses by our framework. We refer to the passage period from "S" to "G" or from "S" to the forcible initialization of the environment as a trial. Fig. 8 shows an example of the transition of the total number of grids which the ship passes through in a trial. We have stopped the algorithm if the ship does not enter the 


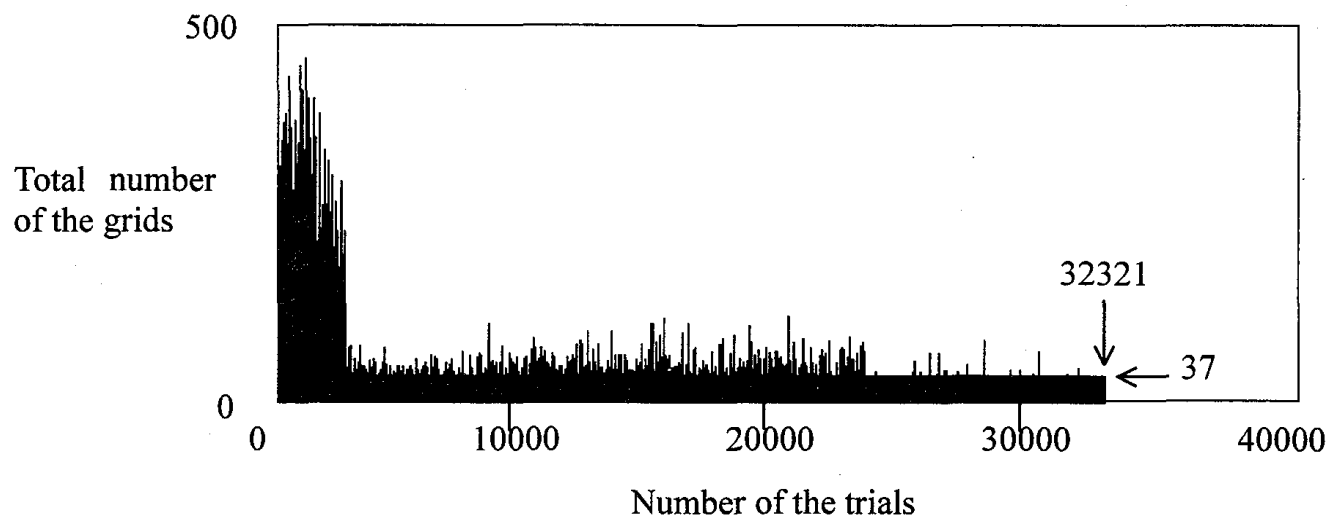

Fig. 8 The transition of the total number of grids which the ship passes through in the situation (a).

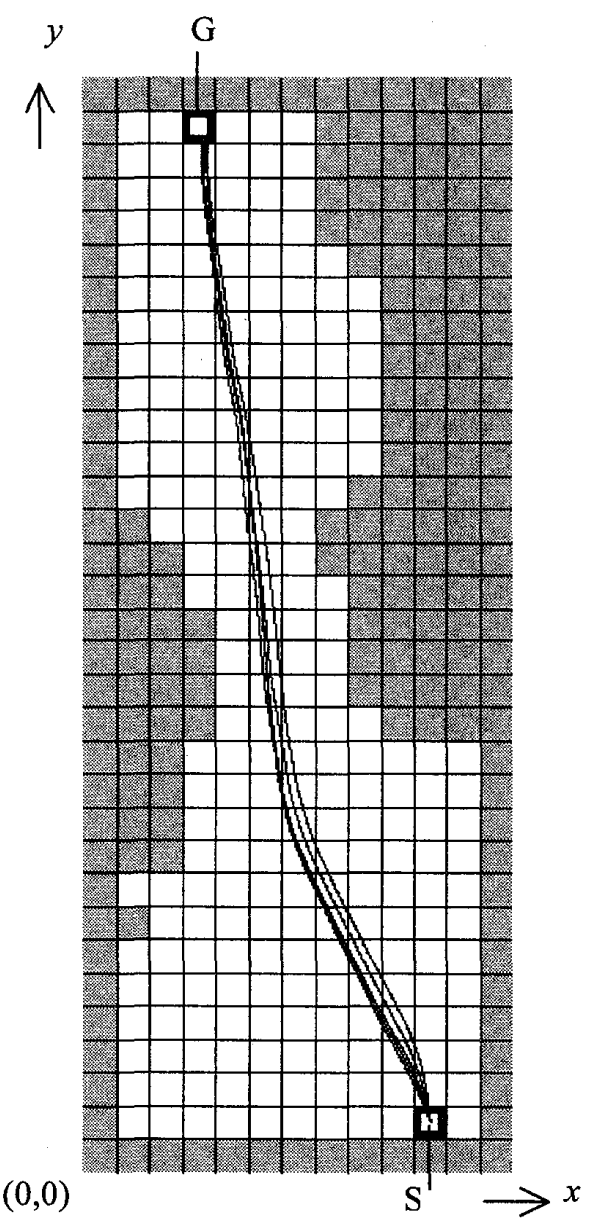

Fig. 9 Several courses in the situation (A) which have been given by applying the greedy policy to $Q(s, a)$ after the stop of the algorithm.

no-entry grid throughout 1000 successive trials after 20000 trials. In this figure, the algorithm was stopped at 32321 trials. Fig. 9 shows several courses given by applying the greedy policy to $Q(s, a)$ after the stop of the algorithm. The ship started with five distinct initial angles included in the range $I_{0}$.

In this problem, the ship is influenced by the following tidal current during the passage from " $\mathrm{S}$ " to

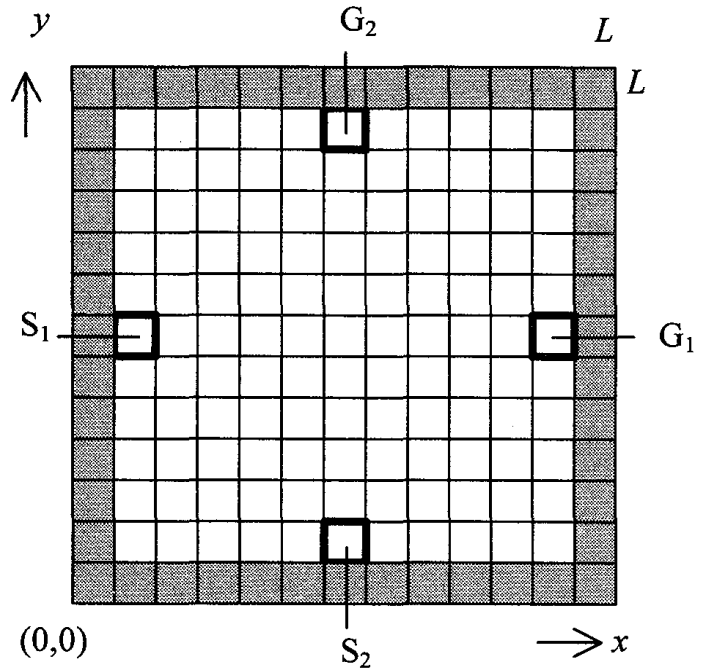

Fig. 10 The model of the sea-area in the situation (B).

"G". The model of sea-area corresponds to the case where the Kurushima strait has the strongest tidal stream. Namely, this problem is one of the hardest realistic problems. From these results, we have confirmed that our framework has sufficiently good performance in such a difficult problem. Thus, we can expect that these results contribute to the indication of the standard pattern of the maneuvering motion and the evaluation of the navigable sea-area.

\section{RL Algorithm to Find the Shortest Course in the Situation (B)}

This section discusses the RL algorithm to find the shortest course in the situation (B) and its calculation results. Fig. 10 shows the model of the sea-area. It has no tidal current. Letting $k$ denote the ship number, the $k$-th ship has the start grid " $\mathrm{S}_{k}$ " and the goal grid " $\mathrm{G}_{k}$ ". If both ships are in a grid at the same time, we regard this situation as "a collision".

We use two agents (i.e., $n=2$ ). Each of them con- 
trols the rudder angle of its ship. The aim of the learning algorithm here is finding the shortest course from " $\mathrm{S}_{k}$ " to " $\mathrm{G}_{k}$ " for each ship without the collision. We apply the $Q$-learning algorithm to this problem according to the following framework:

- The environment for the $k$-th ship is represented by the variables $\left(\phi_{k}, \Phi_{k}, x_{k}, y_{k}\right)$. A point in the space $\left(\phi_{k}, \Phi_{k}, x_{k}, y_{k}\right)$ corresponds to the state $s_{k}$ through the quantization of this space.

- The environment for the $k$-th ship is initialized by setting these variables as follows: $\left(x_{k}, y_{k}\right)$ is set to the center of " $\mathrm{S}_{k}$ ". $\phi_{1}$ and $\phi_{2}$ have the reference directions along $x$ and $y$ axes, respectively. They are set to a common value which is randomly selected from a given initial range $I_{0}$ in order to learn the maneuvering motion to avoid the collision. $\Phi_{k}$ is set to zero. The sub-terminal state is that each ship arrives at its goal grid and the terminal state is that all ships achieve the sub-terminal states.

- In order to select the action number of the $k$-th ship, we employ the $\varepsilon$-greedy policy.

- The movement by the executed action corresponds to 1 time-step of the numerical integration of the motion equations in the rudder angle $\delta_{k}$ specified by $a_{k}$. If the $k$-th ship enters the no-entry grid, we make it escape from the situation according to the same procedures as the section 4 . Also, if both ships are in a grid at the same time, we make them escape from the collision as follows (see Fig.11). If both ships are in a grid at the time $t_{n}$, the four variables $\left(\phi_{k}, \Phi_{k}, x_{k}, y_{k}\right)$ at $t_{n+1}$ are determined by the following procedures.

1. Return each ship to the position $\left(x_{k}, y_{k}\right)$ at $t_{n-1}$.

2. Add $\pi$ to $\phi_{k}$, which makes each ship turn about.

3. Set $\Phi_{k}$ to zero.

- The agent of the $k$-th ship receives the reward $r_{k}$ according to the same rule as the section 4. Also, if both ships are in a grid at the same time, the agents of both ships receive $r_{k}=-1$, but the environment is not initialized.

Furthermore, we fix the ship's forward velocity $V_{0}$, the learning rate $\alpha_{k}$, the initial angular range $I_{0}$, and the probability $\varepsilon_{k}$ at the following values:

$$
\begin{aligned}
& V_{0}=8.6[\mathrm{knots}]=4.42[\mathrm{~m} / \mathrm{s}], \alpha_{1}=\alpha_{2}=0.3, \\
& I_{0}=[-0.15 \pi, 0.0][\mathrm{rad} .], \varepsilon_{1}=\varepsilon_{2}=1.0 \times 10^{-4} .
\end{aligned}
$$

For the quantization of the space $\left(\phi_{k}, \Phi_{k}, x_{k}, y_{k}\right)$, we

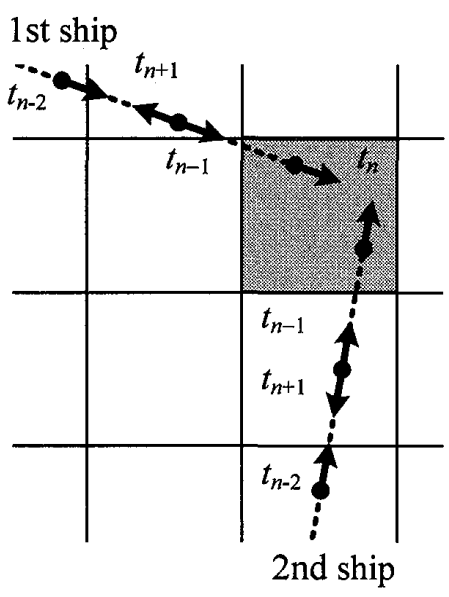

Fig. 11 Escape from the collision.

divide $\phi_{k} \in[0,2 \pi]$ into twelve equal parts and $\Phi_{k}$ into two parts based on its sign. Also, for the division of the subspace $\left(x_{k}, y_{k}\right)$, we make use of the grids. In the model of the sea-area, the total number of the grids is $13 \times 13=169$ and the space $\left(\phi_{k}, \Phi_{k}, x_{k}, y_{k}\right)$ is divided into $12 \times 2 \times 169=4056$-subspaces for each agent. Each of the divided subspaces can be classified by the state number $s_{k} \in S=\{0,1, \ldots, 4055\}$. In the numerical integration, we use the fourth-order Runge-Kutta method. The size of the time-step is $1 / 50$ of the time for the ship to go straight for the distance $L$. For the selection of the rudder angle $\delta_{k}$ [deg.], we prepare the seven kinds of the values $\{0,2.5,-2.5,5,-5,10,-10\}$ and they correspond to the action numbers $\{0,1,2,3,4,5$, $6\}$, respectively.

Next, we show the calculation results of the courses by our framework. A trial means that all the agents achieve the sub-terminal states once. Fig. 12 shows an example of the transition of the total number of grids which each ship passes through in a trial. We have stopped the algorithm if both ships do not enter the no-entry grid and collide throughout 1000 successive trials after 20000 trials. In this figure, the algorithm was stopped at 29264 trials. Fig. 13 shows several courses which have been given by applying the greedy policy to $Q_{k}\left(s_{k}, a_{k}\right)$ after the stop of the algorithm. Fig. 13(a) shows one of them. In this figure, the courses of the 1st ship and the 2nd ship are emphasized every 50 time-steps of the numerical integration by the black and white squares, respectively. The 1st ship goes along the curved course while the 2 nd ship goes along the almost straight course. Although there is a grid which has an intersection between these courses, the grid includes the fourth black square and the fifth 
100

Total number of the grids of 1 st ship

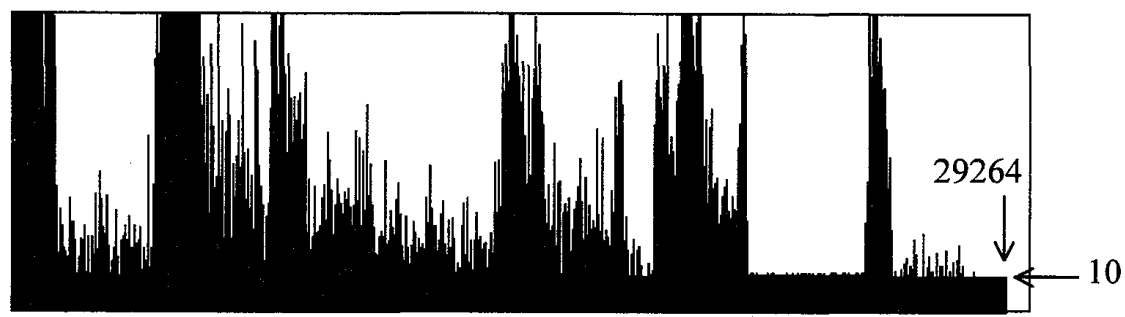

Total number of the grids of 2nd ship

0

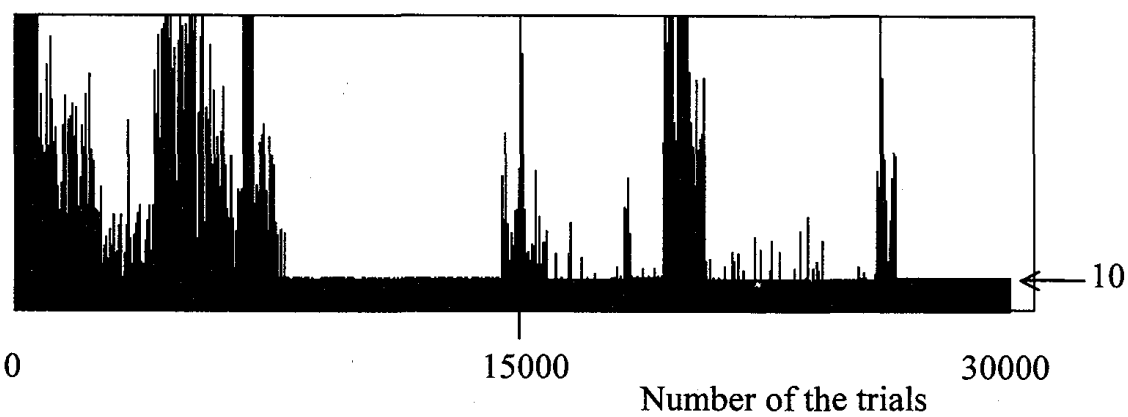

Fig. 12 The transition of the total number of grids which the ship passes through in the situation (B).

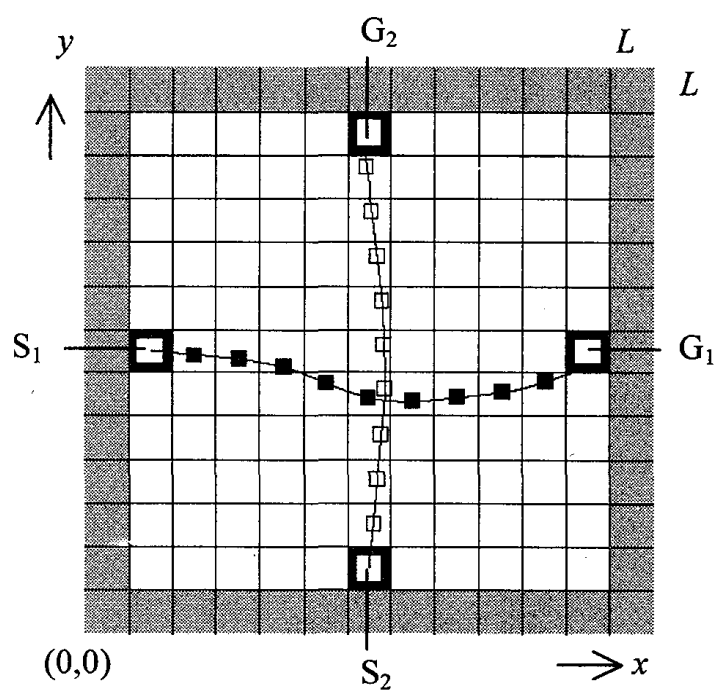

(a)

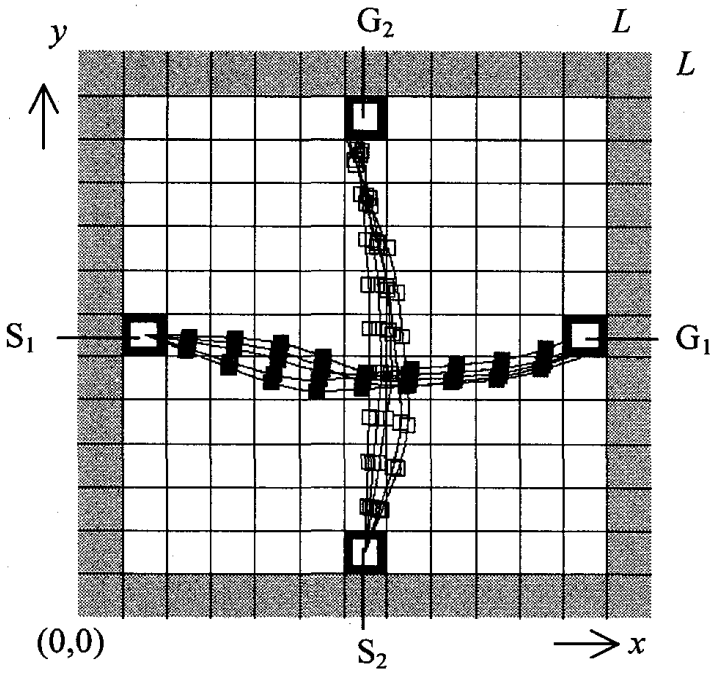

(b)

Fig. 13 Several courses in the situation (B) which have been given by applying the greedy policy to $Q_{k}\left(s_{k}, a_{k}\right)$ after the stop of the algorithm: (a) an initial angle and (b) five distinct initial angles.

white square. This result means that "both ships are not in the common grid at the same time, that is to say, the collision between them is avoided". In Fig. 13(b), the ships started with five distinct initial angles included in the range $I_{0}$.

Though the crossing situation rule in COLREG is not embedded in this algorithm, we have derived the pattern of the maneuvering motion which is often observed in the actual navigations as shown in Fig. 13. From these results, we have confirmed that our framework has sufficiently good performance in this problem. The rule to avoid the collision between two ships is determined in COLREG. These calculation results may contribute to the construction of the rule to avoid the collision between more than two ships.

\section{Conclusion}

We have constructed the RL framework to find the shortest course in two fundamental situations. The effectiveness of this framework has been demonstrated with the model of the sea-area. Our future problems can be enumerated as follows:

I) Although we treat finding the shortest course in each of the situations as an individual fundamental problem, the combination of them provides more realistic problem. Since the learning algorithm can 
be applied to such a problem, the result may grow into a novel simulation technique of the traffic flow on the sea which reflects both the tidal current effect and the interaction between the maneuvering actions of the navigators.

II) We have employed KT model with the tidal current effect for the sake of simplicity. It cannot sufficiently describe the situation where the ship speeds up and down suddenly. We should also discuss the RL algorithm using more detailed model of maneuvering motion of the ship.

III) These realistic and detailed approaches require very high computational cost. Therefore, we should improve the learning algorithm so that it will provide the efficient and sure learning process, by introducing the hierarchical structure of $Q^{(8)}$, the order of the priority in the competition between the agents ${ }^{(9)}$, and so on.

IV) We have applied the fixed division to the space of the variables in the motion equations and have adjusted the number of the division by trial and error to achieve the sufficient learning performance. In view of the efficiency, the adaptive division of the space is more desirable than the fixed division. Now we are considering the adaptive division that can be combined with our framework.

\section{Acknowledgements}

The authors would like to thank Prof. $\mathrm{H}$. Hinata in Japan Coast Guard Academy for his helpful discussions and suggestions about the equations of the maneuvering motion of a ship, and Prof. N. Suematsu in Hiroshima City University for his valuable discussions about the RL algorithm.

\section{References}

(1) R.L.Sutton and A.G.Barto, "Reinforcement learning: an introduction", The MT Press, Cambridge, MA (1998). This book is translated into Japanese and published as the following one: S.Mikami, and M.Minagawa, "Kyouka Gakusyuu", Morikita Syuppan, Tokyo (2000).

(2) M.L.Littman, "Markov games as a framework for multi-agent reinforcement learning", in Proc. of $11^{\text {th }}$ International Conference on Machine Learning, pp. 157-163 (1994).

(3) T.Horiuchi, A.Fujino, O.Katai and T.Sawaragi, "Q-PSP learning: an exploitation-oriented $Q$-Learning algorithm and its applications", Trans. of Society of Instrument and Control engineers, vol.35, no.5, pp.645-653 (1999). (in Japanese)

(4) S.Arai, "Multi-agent Reinforcement Learning Frameworks: Step Toward Practical Use", Journal of Japanese Society for Artificial Intelligence, vol.16, no.4, pp.476-481 (2001). (in Japanese)

(5) C.J.C.H.Watkins and P.Dayan, " $Q$-Learning", Machine Learning, 8, pp.279-292 (1992).

(6) K.Honda, "Theory to maneuver a ship", Seizan-dou, (1995). (in Japanese)

(7) Japan Coast Guard Agency, "Charts of tidal streams in Kurushima Kaikyo", Sheet no.10, (1990).

(8) T.G.Dietterich, "Hierarchical reinforcement learning with the MAXQ value decomposition", Journal of Artificial Intelligence Research, 13, pp.227-303 (2000).

(9) R.Sun and C.Sessions, "Self-segmentation of sequences: automatic formation of hierarchies of sequential behaviors", IEEE Trans. on Systems, Man, and Cybernetics: Part B, Cybernetics, vol.30, no.3, pp.403-418 (2000).

\section{Question \& Answer}

Question: Masayoshi Numano (National Maritime Research Institute)

In your algorithm, the reward depends on the grid in which the ship is right after the execution of the action. Generally speaking, if the ship avoids the collision with the obstacle, it must make a decision to change the course before they are close to each other. Is this fact reflected in your algorithm?

\section{Answer: Kunihiko Mitsubori}

It is reflected in our algorithm, because the increase and decrease of the value of the action is spatially propagated as the trial is iterated. While the number of the trials is small, the action to avoid the collision is learned in the grid that neighbors on the obstacle. As the number of the trials increases, such action is learned in the grid that is a long way from the obstacle. That is to say, a sequence of the optimal actions is determined one by one from the action in the grid that is close to the goal grid. Therefore, finding the optimal requires the sufficient repeat of the trials. 